\title{
Prevalence of hypotension and its association with cognitive function among older adults
}

\begin{abstract}
Background and Objective: The negative effect of hypertension has overshadowed possible health problems associated with hypotension. The purposes of this study were to describe the prevalence of hypotension in older adults and to determine the association between hypotension and cognitive function, after adjusting for possible covariates. Methodology: The data for the study consisting of 1067 community-dwelling older adults were obtained from a national survey entitled "Identifying Psycho social and Identifying Economic Risk Factor of Cognitive Impairment among Elderly", conducted in Malaysia. The hypotension was considered as blood pressure $<120 / 75 \mathrm{~mm} \mathrm{Hg}$, measuring by standard mercury manometer. Data analysis was performed using the SPSS Version 22.0.Results: The mean age of the respondents was 68.27 (SDD5.93). Mean score of cognitive function as measured by MMSE was 22.70 (SDD4.95). The prevalence of hypotension was 29.3\%. The prevalence of cognitive impairment for hypotension group was $25.6 \%$. Results of multiple linear regression analysis revealed that hypotension is negatively associated with cognitive function (BetaD $; 0.11, \mathrm{p}<.01)$, after adjusting for age, gender, education, marital status, employment status, diabetes, heart disease, stroke and gastritis. Conclusion: The study showing hypotension is significantly associated with decreased cognitive function in later life, implies more attention to low blood pressure in old age.
\end{abstract}

Keyword: Aged; Cognition; Hypotension 